\title{
Full PON Virtulisation Supporting Multi-Tenancy Beyond 5G
}

\author{
Nima Afraz, Frank Slyne and Marco Ruffini \\ CONNECT Center, Trinity College Dublin, Ireland \\ \{nafraz, fslyne, marco.ruffini\}@tcd.ie
}

\begin{abstract}
In this paper, we introduce a virtualization technique to enable fully customizable resource sharing for Passive Optical Networks. We provide a summary of the concept, economic challenges and implementation. (C) 2019 The Author(s)
\end{abstract}

OCIS codes: 060.4250 Networks; 060.4256 Networks, network optimization.

\section{Introduction}

The current scene on the broadband/mobile operators' market is an oligopoly, where novelty is limited by the market development policies of a hand-full of operators. The cost of entering this market is unaffordably high for smaller service providers who could bring considerable revenue to the access market by introducing new services. Sharing the last mile of access networks, which is the most CAPEX demanding part, can dramatically reduce the required initial investment and facilitate market entrance for new operators. However, the current sharing methods, especially in fixed access networks, operate at too a high-level (e.g., virtual unbundled local access (VULA)) where they are not capable of providing enough control over the service provided to the customers [1]. Other proposals exists for a low-level access, which typically translate in assigning a dedicated wavelength to a second operator. However, besides being inefficient, they are currently hindered by the fact that multi-wavelength PON (e.g., NGPON2) has not been deployed anywhere due to its high cost. Therefore, we propose a new sharing technique for Passive Optical Networks (PONs) which meets the above-mentioned methods in the halfway by providing framelevel scheduling control for the operators while being more affordable and easier to attract new entrants [2]. In the next section, we will elaborate on the importance of providing scheduling control for the operators. Then we will slightly touch on the economic market challenges in multi-tenant fixed access sharing environments and finally share some details about the test-bed implementation of the proposed sharing method.

\section{The Sharing Model}

Due to their worldwide deployment and efficiency of resource usage, PON networks are considered a strong candidate for providing networks' services to 5G networks and beyond [3]. However, the capability of the current PON networks to support new services with stringent and diverse requirements such as latency has been a center of focus for a large and growing body of literature.

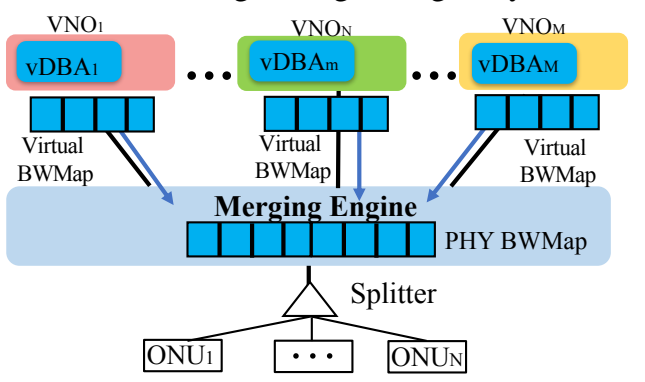

Fig. 1. Multi-Tenant PON Layout with vDBA

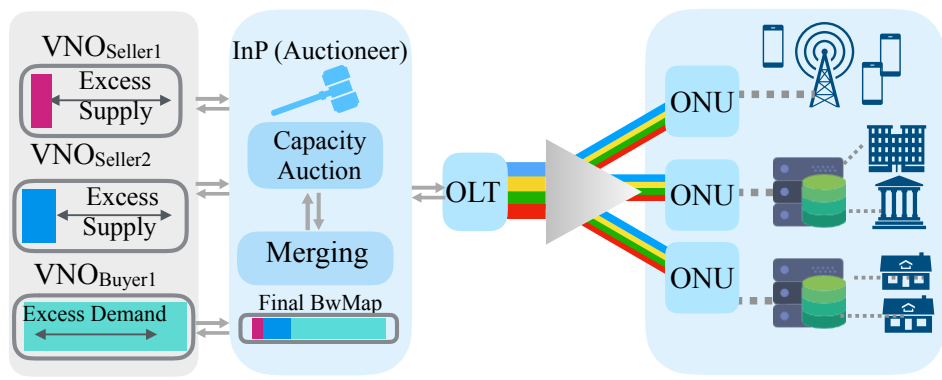

Fig. 2. Multi-Tenant PON Sharing Market

However in the upstream, conventional PON scheduling schemes, called Dynamic Bandwidth Allocation (DBA), are incapable of supporting low latency as it operates extensive and time-consuming signaling between the OLT and the ONUs. Our proposed virtualisation of the DBA (vDBA), recently standardised in the BroadBand Forum (BBF) TR-402 and depicted in Figure 1, allows different Virtual Network Operators (VNOs) to implement their flavour of the DBA, providing them with the required flexibility to control the upstream scheduling, paving the way for adoption of PONs as primary transport network solution for new bandwidth-intensive services (5G, Virtual reality etc.). In [4] we have addressed the possibility of providing the required control to the VNOs by dedicating virtual and programmable instances of the DBA algorithms. Therefore, each VNO will operate a portion of the network and their bandwidth allocation decision (referred to as bandwidth map (BwMap)) is aggregated by the merging engine in the final BwMap is issued. The technical details of this work are further discussed in subsection 3 . 
One issue that arises once the scheduling is passed to VNOs is that without proper incentives, VNOs have little motivation in sharing any unused capacity with other VNOs, making the overall PON inefficient. Indeed, once VNOs can fully control their slice scheduling their best strategy is to always pretend they need all the contracted capacity, which might otherwise be re-directed to a competing VNO. To address this challenge, we have proposed [5] monetization of the excess PON slices where using an auction mechanism the VNOs can trade their excess capacity in return for monetary compensation (depicted in Figure 2). Through rigorous theoretical proofs and market simulations in [6] we validate that the proposed market will incentivize the VNOs to trade their excess resources in a setting where any attempt to manipulate the market will lead to worst or the same outcome. Nonetheless, the previous work assumes an open access architecture where a fully trusted central authority (Infrastructure Provider (InP)) is in charge of operating the market (bookkeeping, conducting the auction, settlements, etc.). This assumption may not be valid considering the new network ownership models. Hence, the natural progression of this work is to study distributed means of consensus (e.g., Blockchain-based Smart Contracts) which do not rely on a central entity.

\section{Implementation}

Our vDBA concept was demonstrated in [7] on test bed incorporating a physical PON, a set of emulated ONUs, a traffic generator and a multi-access edge computing node. The physical PON was implemented on Xilinx VC709 FPGA boards, operating at symmetric $10 \mathrm{~Gb} / \mathrm{s}$ line rate.

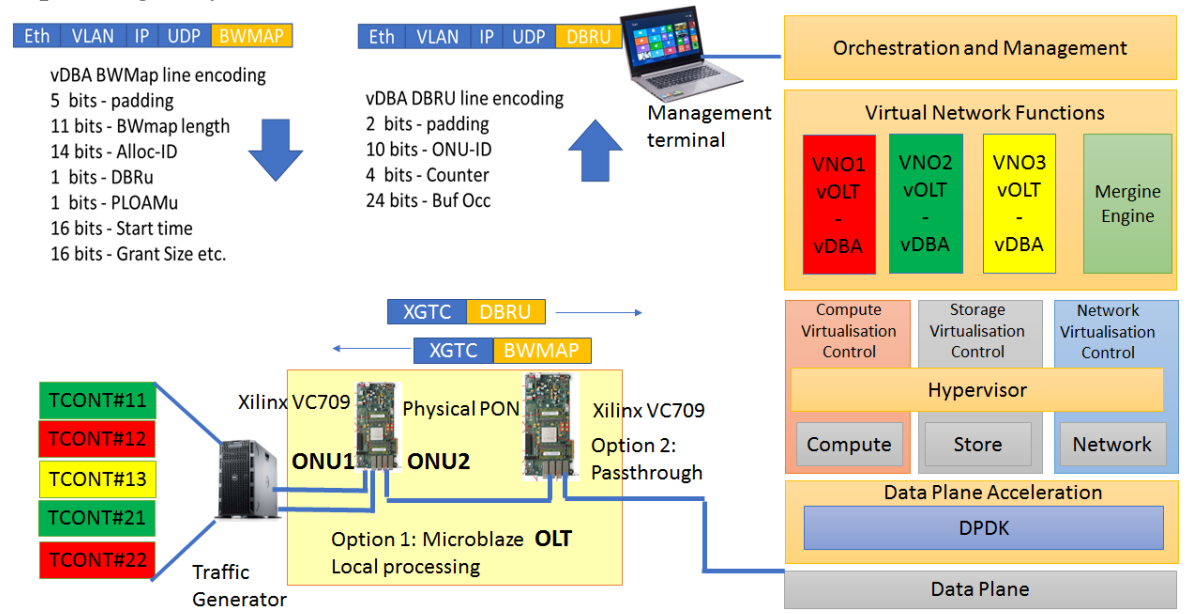

Fig. 3. vDBA edge Compute stack

The PON hardware and software virtualisation architecture is shown in Fig. 3. The Multi-access Edge Computing (MEC) node hosted the Merging Engine (ME) and the vDBA functions for the Virtual Network Operators (VNOs), implemented as Virtual Network Functions. The Merging Engine is the element that blends together all virtual bandwidth maps from the different VNOs so as to generate one physical bandwidth Map allocation) and the SDN control plane. Due to the real-time critical nature of receiving and transmitting status report messages from the ONUs (called DBRUs) and Bandwidth Map data, our latest implementation [8], [9] made advanced use of the Data Plane Development Kit (DPDK) toolkit to optimize the packet transfer through the physical host to and from the Virtual Network Functions.

\section{References}

1. M. Ruffini, Multidimensional convergence in future 5g networks, JLT, 35 (3), Feb. 2017.

2. A. Elrasad et al., Virtual dynamic bandwidth allocation enabling true PON multi-tenancy, OFC 2017, paper M3I.3.

3. J. S. Wey et al., Passive optical networks for 5g transport: Technology and standards, JLT, July 2018.

4. A. Elrasad et al., Frame level sharing for DBA virtualization in multi-tenant PONs, ONDM 2017.

5. N. Afraz et al., DBA capacity auctions to enhance resource sharing across virtual network operators inmultitenant PONs. OFC 2018, paper Th1B.3.6.

6. , A sharing platform for multi-tenant pons, JLT, 36 (23) Dec 2018.

7. F. Slyne et al., Demonstration of real time VNF implementation of OLT with virtual DBA for sliceable multi-tenant PONs, OFC 2018, papaer Tu3D.4.

8. F. Slyne et al., Experimental demonstration of DPDK optimised VNF implementation of virtual DBA in a multi-tenant PON ECOC 2018.

9. M. Ruffini et al., Moving the network to the cloud: The cloud central office revolution and its implications for the optical layer. JLT 37 (7), April 2019. 